\title{
Desmoplastic Soft Tissue Tumor- A Case Report
}

\author{
SHORMIN ARA FERDOUSI ${ }^{1}$, AFROZAAKHTER ${ }^{2}$, WASIM SELIMUL HAQUE ${ }^{3}$, AFIQUL ISLAM $^{4}$
}

\section{Introduction}

Desmoplastic small round cell tumor (DSRCT) is a rare highly aggressive malignancy, commonly presenting as a large intra-abdominal or pelvic mass in male children and young adults. Microscopically, the tumor is typically composed of discrete nests of primitive round cells surrounded by a prominent desmoplastic stroma. Extra-abdominal cases, though limited, have been reported in several locations: mediastinum, head and neck region, central nervous system, paratesticular region, visceral organs, and soft tissue. Infrequently, DSRCTs may present with bony metastasis. ${ }^{1}$ It was first described by Gerald and Rosai in 1989 and formally named in 1991. The histogenesis of this tumor remains unclear but might be derived from multi potential differentiated primitive mesenchymal cells or neuroectodermal and primitive mesenchymal tissue. ${ }^{2}$ DSRCT mainly develops in adolescent and young adults with a strong male predominance; the mean age at diagnosis is approximately 22 years and ranges from 6 to 49 years, the male to female ratio is $4: 1 .^{3}$ This case is an adolescent boy who presented with abdominal symptoms.

\section{Case Report}

An11 year's male child admitted with the complaints of nonspecific abdominal pain for one month which was dull aching aggravated at night not associated with vomiting, loose stool, and fever and not relieved by taking food or increase in empty stomach. He had also complaints of dysphasia in taking solid food for fifteen days. On examination he was afebrile, mildly anictenic vitals are within normal limits, there was no lymhadenopathy, and BCG mark was present. Abdomen was soft, flank empty, diffusely tender more in epigastric region, ill-defined mass about $4 \times 3 \mathrm{~cm}$ located in epigastric and right hypochondriac region which was non-tender, mobile and firm. There was no

1. Lt. Col. Classified Child Specialist \& Pediatric Oncologist, Combined Military Hospital, Dhaka

2. Classified Child Specialist, Combined Military Hospital, Dhaka

3. Classified Pathologist, Armed Forces Institute of Pathology, Dhaka

4. Professor of Pediatric Hematology and Oncology, BSMMU, Dhaka

Correspondence : Dr. Shormin Ara Ferdousi, E-mail: shormin@ gmail.com organomegaly. His Complete Blood Count (CBC), all serological and biochemical profile were within normal limit except LDH which was 1255 u/l. $\beta$-fetoprotein, CEA, $\beta$-HCG were also within normal limit.

USG of abdomen showed a large lobulated mass measuring $9.2 \times 3.3 \mathrm{~cm}$ in retroperitoneal region, compressing the pancreas anteriorly and encasing the abdominal aorta \& Inferior Vena Cava and ascites present.

CT Abdomen (Fig.-1) revealed multiple lobular \& matted structures in the para-aortic and pericaval region extending throughout the whole length of abdominal aorta. Celiac trunk, superior, Inferior, mesenteric \& renal arteries along with aorta are encased by the lobulated soft tissue mass. Inferior Vena Cava appears compressed and pushed anterolaterally and minimum ascites was present, commented as extensive abdominal lymphadenopathy with minimal ascites and lymphoma was the possibility.

CT guided FNAC from abdominal mass showed highly cellular smears composed of small round cells arranged diffusely. The individual cells have scanty cytoplasm. A few of the cells showed small clustering which was consistent with Small round blue cell tumor.

Biopsy by diagnostic laparotomy followed by Histopathological Examination (Fig.-3) revealed a malignant neoplasm composed of sheets of undifferentiated cells separated by fibrous stroma. The tumor cells have scanty cytoplasm and inconspicuous nucleoli. Tiny foci of necrosis were present.

Immunohistochemistry showed cytoplasmic strongly positive (4+) for desminand weakly positivity $(2+)$ for Neuron Specific Enolase (NSE) and CD56 and poorly positive (1+) for CD99 and Cytokeratin (CK).

The morphology, immune histo chemistry markers were consistent with the diagnosis of DSRCT. Due to unavailability, cytogenetic study was not done.

CT chest showed ground glass density in medial segment of right middle lobe, suggestive of inflammatory lesion in right side with mild bilateral pleural effusion.

Plan of treatment was chemotherapy with EpSSG NRSTS 2005 (protocol P6) followed by surgical 


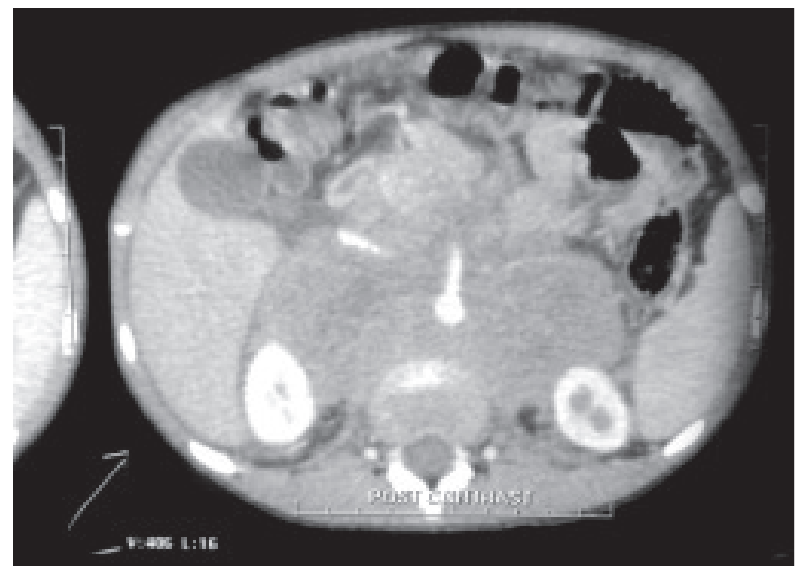

Fig.-1: CT abdomen at presentation

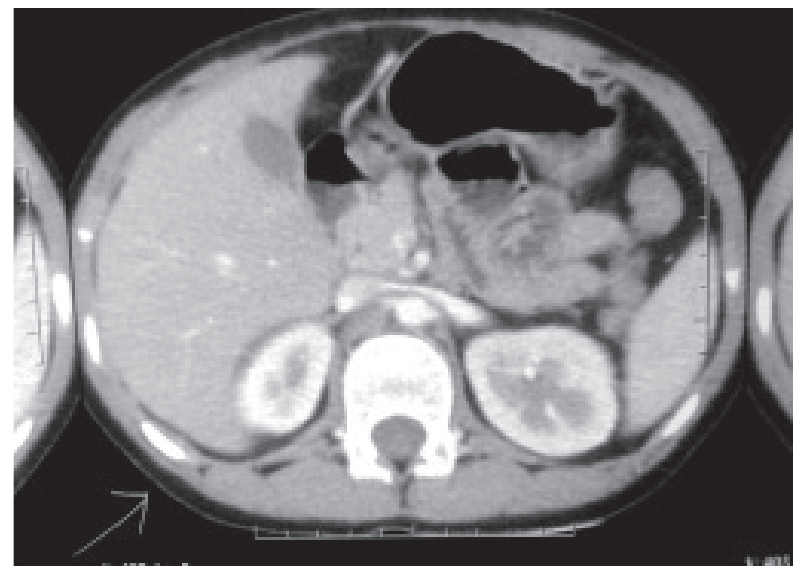

Fig.-2: CT abdomen after completion Chemotherapy

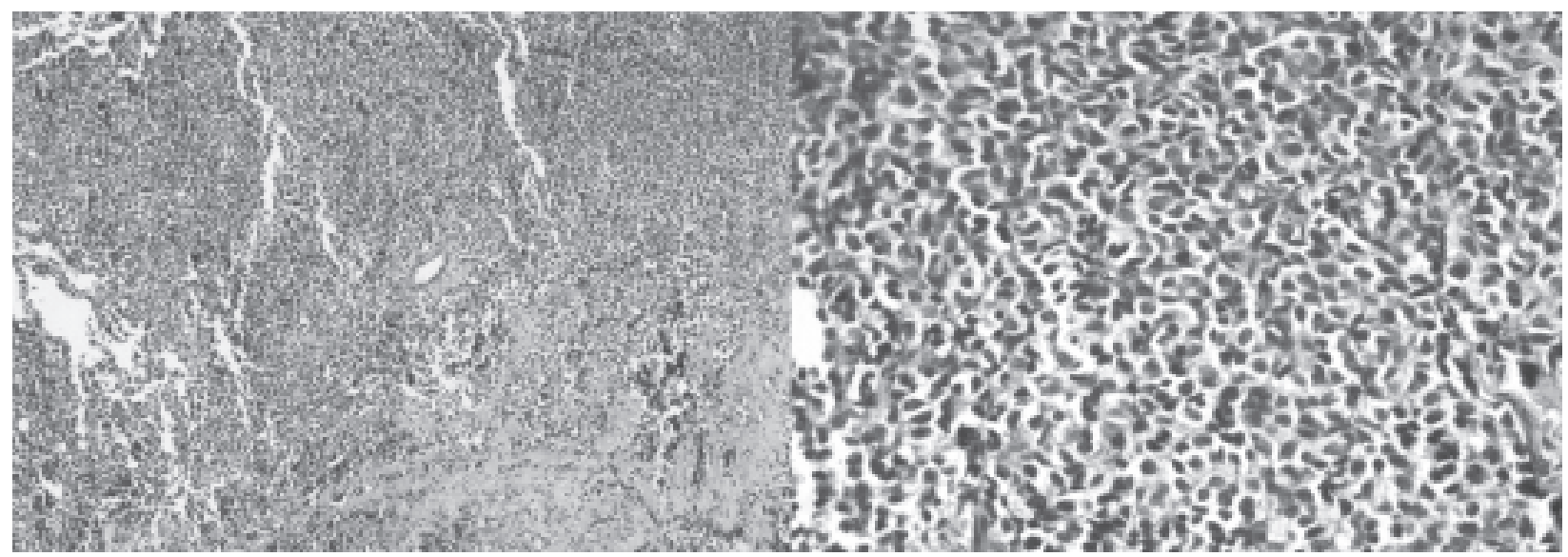

Fig.-3: Histological findings of desmoplastic small round cell tumor

excision after 4-6 cycle of chemotherapy then Radiotherapy. Chemotherapy started with etoposide, ifosphamide, cyclophosphamide, doxorubicin and vincristine. After 3rd cycle of chemotherapy repeat CT abdomen (Fig:2) and chest showed no residual mass, so surgical excision was not needed and chemotherapy continued. He completed total 07 cycle of chemotherapy. Now he is waiting for radiotherapy. $\mathrm{He}$ is stable and on regular follow-up.

\section{Discussion}

DSRCT belongs to the family of "round blue cell tumors" commonly found in the pediatric population. Round blue cell tumor includes neuroblastoma, malignant lymphoma, rhabdomyosarcoma, Ewing's sarcoma, Wilms' tumor, and primitive neuroectodermal tumor (PNET). ${ }^{4}$ DSRCT is an extremely rare and aggressive soft tissue sarcoma, with only a few hundred cases reported in the literature. The etiology of DSRCT is unknown but it is thought to be a malignancy of the mesothelium. ${ }^{5} \mathrm{As}$ it can have delitescent onset without apparent symptoms and is highly aggressive, DSRCT is often diagnosed at a relatively advanced stage. The diagnosis of DSRCT remains challenging. Because most DSRCTs are found in the abdominal cavity, some non-specific gastrointestinal symptoms (such as nausea, vomiting, abdominal distension and pain) and a palpable abdominal mass are the main problems leading to patients visiting a doctor. If present in extraperitoneal sites, DSRCT can cause specific symptoms, mostly owing to expansion and compression of the tumor. Weight loss, acratia, ascites, and cachexia may occur when there is tumor dissemination or extensive lymphogenous and hematological metastases. ${ }^{6}$ Our patient had abdominal pain with dysphagia and there was a ill-defined abdominal mass at presentation without any metastasis and his general condition was good.

DSRCT is associated with a distinct translocation which shows a fusion between Ewing sarcoma gene 
(EWSR1) and Wilms tumor gene (WT1). There are at least two reports of hybrid tumors with features of both DSRCT and Ewing sarcoma, one with an EWSR1FLI1 fusion gene and one with an EWSR1-ERG fusion gene. ${ }^{7}$ Due to unavailability of cytogenetics in our country by FISH method we could not find out the translocation in our patient. Clinically, patients present symptoms of abdominal sarcomatosis such as ascites, abdominal pain and/or distension, constipation or bowel obstruction, vomiting, and weight loss.

Abdominal imaging by ultrasound, computed tomography scan or magnetic resonance imaging reveals multiple peritoneal masses (from millimeter sized nodules to confluent sheets and dozens to hundreds of nodules up to $20 \mathrm{~cm}$ or greater). Since it may metastasize to the liver, most commonly, and lungs, and or lymph nodes of the groin, neck, or mediastinum, once abdominal imaging reveals multiple tumors, imaging of the chest with a CT scan and distant metastasis using a total body positron emission tomography (PET) scan, is recommended as the preferred. ${ }^{2}$

Due to some limitations we, in our country prefer FNAC which usually make a diagnostic dilemma like in the case reported by Morshed AKMAet al ${ }^{8}$. Percutaneous or open biopsy of the lesion should be evaluated by immune histochemistry and cytogenetics to confirm the characteristics of DSRCT. ${ }^{2}$ The diagnosis can be confirmed by histological and immune histochemistry studies. ${ }^{3,9}$ The typical histology includes variably sized clusters of small round or spindled cells lying within a desmoplastic, collagenous stroma. In most cases of DSRCT desmin, CK EMA, and vimentinare positive, of which desmin and CK being positive at the same time is considered DSRCT's most specific immunological indexes. ${ }^{2}$

There is no standard protocol for the treatment of the disease. Current therapies are mainly dependent on surgical resection, chemotherapy, and radiotherapy. ${ }^{2}$ However, recent literature and studies have reported that some patients respond to high-dose chemotherapy with other modalities of treatment such as tumor debulking, cyto reductive surgery, and radiation therapy with stem cell rescue. According to the patient's condition, interventional therapy can be given, and preoperatively induced chemotherapy, surgery, and radiotherapy. Recent studies show that complete surgical excision, including a 1 to $2 \mathrm{~mm}$ long implant, is necessary to achieve long-term disease control. Intraperitoneal hyperthermic chemotherapy using cisplatin is thought to be effective at reducing the recurrence rate of disease. Overall the prognosis for DSRCT is poor with less than $20 \%$ surviving beyond two to three years. ${ }^{10-12}$

\section{Conclusion}

DSRCT is a rare malignant tumor with poor prognosis. Despite its rarity, several new procedures have been tested in DSRCT. The diagnosis is usually established by immune histochemistry. To date, no standard treatment protocol has been proposed.

\section{References}

1. Khachaturov V, Christopher RW, Jacob R, Hodge JR, Doyle JJ, Leitao DL et al. Primary intraosseousdesmoplastic small round cell tumor of the calvarium: Case report and review of the literature. Human Pathology. 2015;2:9-15.

2. Xiang L, Jing $Y$, Shibao F, Xiaoming $X$, Jie $Z$. Desmoplastic small round cell tumor: a case report and review of the literature. World Journal of Surgical Oncology. 2014;12:1-4.

3. Armelle D, Philippe C, Laure C, Perrine M, Pierre M, Laurent A et al. Desmoplastic Small Round Cell Tumor: Current Management and Recent Findings. Sarcoma. 2012;ID 714986;1-5.

4. Richard B, Lisa Suzuki B, Eric B, Jill PG, Maris J, Bruce RP. Desmoplastic Small Round CellTumor in the Abdomen and Pelvis:Report of CT Findings in 11 Affected Children and Young Adults. American Journal of Roentgen Ray Society. 2005;184:1910-14.

5. Robert T, Gajan R, Khin T, Charlotte B, Khawaja $\mathrm{S}$, Eleanor M. Desmoplastic small round cell tumour: The Radiological, Pathological and Clinical features. Insights Imaging. 2013;4: 111-18.

6. Gui MZ, Yao Z, Hua LG, Ding WY. Testicular desmoplastic small round cell tumor: a Case Report and Review of Literature. World Journal of Surgical Oncology. 2014;12:1-4.

7. Vincent PMC, Károly S, Hans JB, Jurriaan VD, Hans G, Pancras CWH. Paratesticular Desmoplastic Small Round Cell Tumour: an Unusual Tumour with an Unusual Fusion; Cytogenetic and Molecular Genetic Analysis Combining RT-PCR and COBRA-FISH. Clinical Sarcoma Research. 2012;2:1-7. 
8. Morshed AKMA, Afroz F, Galib CMF, Islam S, Hasina K, Ghosh AK. Desmoplastic Small Round Cell Tumor (DSRCT)-A Case Report and Review of Literature. Journal of Paediatric Surgeons of Bangladesh. 2011;2:79-82.

9. Guangzhao Z, Guangjun L, Dahua Z, Xijun C, Gang L. Desmoplastic Small Round Cell Tumor of the Abdomen and Pelvis: Clinico-pathological Characters of 12 Cases Guangzhao. Scientific World Journal. 2014;ID 549612:1-8.

10. Christina KL, Pamela GF, Pooja H. Incidence and Outcomes of Desmoplastic Small RoundCell Tumor: Results from the Surveillance,
Epidemiology, and End Results Database. Journal of Cancer Epidemiology. 2014;ID 680126:1-5.

11. Preethika A, Khalafallah AA, Karen W, David S, Terry B. An Unusual Presentation of Desmoplastic Small Round Cell Tumour of the Abdomen: Morphological, Immunohistochemical, Ultrastructural, and Molecular Studies. Case Reports in Oncological Medicine. 2011;ID 183938:1-4.

12. Andrea $\mathrm{nHJ}$, Peter MA. The Diagnosis and Management of Desmoplastic Small Round Cell Tumor: a review. Curr Opin Oncol. 2011;23: 385-89. 